\title{
Antimicrobial Susceptibility in Intensive Care Units: MYSTIC Program Brazil 2002
}

Caio Mendes ${ }^{1}$, Carmen Oplustil ${ }^{1}$, Elsa Sakagami ${ }^{1}$, Philip Turner ${ }^{2}$, Carlos Kiffer ${ }^{1}$, and the MYSTIC Brazil Group

\begin{abstract}
Fleury Institute, Advisory Group on Antimicrobials and Clinical Microbiology ${ }^{l}$, São Paulo, SP, Brazil; AstraZeneca Pharmaceuticals ${ }^{2}$, Macclesfield, Cheshire, England
\end{abstract}

Objective. Establish the susceptibility pattern of Gram-negative bacteria causing infections in ICU patients, MYSTIC Program Brazil 2002. Material and Methods. Gram-negative bacteria $(n=$ 503) causing nosocomial infections were collected at seven Brazilian centers. The central laboratory confirmed the identification and performed the susceptibility tests by E-test methodology (AB Biodisk, Solna, Sweden) for meropenem, imipenem, ciprofloxacin, ceftazidime, cefepime, cefotaxime, piperacillin/tazobactam, gentamicin, and tobramycin. Interpretation criteria used were according to National Committee for Clinical Laboratory Standards (NCCLS). Results. Pseudomonas aeruginosa (33\%) was the most frequently isolated, followed by $\mathrm{A}$. baumannii (17.1\%), K. pneumoniae (12.1\%), E. coli $(10.5 \%)$, and E. cloacae $(7.9 \%)$. Pseudomonas aeruginosa isolates had susceptibility rates of $67.5 \%$ to piperacillin/tazobactam, $59.8 \%$ to meropenem, $57.3 \%$ to imipenem. A. baumannii presented susceptibility rates to meropenem of $\mathbf{8 9 . 5} \%, \mathbf{8 8 . 4 \%}$ to imipenem, and $74.4 \%$ to tobramycin. $E$. coli and $K$. pneumoniae were fully susceptible to both carbapenems. Conclusions. Carbapenem resistance among Enterobacteriaceae is still rare in this region. A. baumannii and P. aeruginosa presented elevated resistance rates to all antimicrobials. Since these two bacterial species play an important role in nosocomial infections, the use of empirical combination therapy to treat these pathogens may be justified.

Key Words: Drug resistance, bacterial, microbial sensitivity tests, infection control, carbapenems.

Antimicrobial resistance among pathogens causing hospital-acquired infections is a major worldwide issue, which must be dealt with continuously [1-4]. Surveillance programs are valuable tools and offer important information on bacterial resistance trends, by geographical location and by disease type in community and hospital settings. Several studies have reported higher rates of antimicrobial resistance among isolates from intensive care units (ICUs) than among

Received on 13 July 2004; revised 28 November 2004.

Address for correspondence: Dr. Carlos R.V. Kiffer, M.D., Ph.D. Fleury Institute / Setor de Microbiologia. Avenida General Waldomiro de Lima 508, Zip code: 04344-070 - Jabaquara São Paulo - SP, Brazil. Phone/Fax: +55 115014 7601. E-mail: carlos.kiffer@fleury.com.br

The Brazilian Journal of Infectious Diseases 2005;9(1):44-51 (C) 2005 by The Brazilian Journal of Infectious Diseases and Contexto Publishing. All rights reserved. isolates from general patient-care areas [1,57]. Consequently, proper surveillance programs focused on specific patient-care areas have become a focal point in combating the development of resistant organisms [3-5,7-13]. Furthermore, information on the minimum inhibitory concentration (MIC) generated by such programs help guide antimicrobial therapy before susceptibility tests are available, and they may help prevent the overuse of certain compounds [9]. However, surveillance programs are limited in their ability to address all relevant clinical and microbiological outcome issues. Thus, efforts must be made to better understand bacterial resistance trends and to refine clinical decision tools locally. The Meropenem Yearly Susceptibility Test Information Collection (MYSTIC) program is a global, annual and multicenter surveillance study that compares the activity of several broadspectrum antimicrobial agents in carbapenem user 
centers. These data could be used, in conjunction with other related studies, to properly interpret significant resistance patterns and choose the most appropriate antimicrobial regimens for empirical therapy.

The objective of our study was to determine the susceptibility pattern of Gram-negative bacteria causing nosocomial infections in ICU patients, as part of the third edition of MYSTIC Program Brazil during 2002.

\section{Material and Methods}

Full details of the study design and susceptibility testing methods have been previously described $[14,15]$.

\section{$\underline{\text { Participating centers }}$}

All isolates were collected during 2002 from hospitalized patients form seven ICUs in four Brazilian cities. Centers 1, 4, 6, 7 were located in São Paulo, center 2 in Florianópolis, center 3 in Rio de Janeiro, and center 5 in Brasília (Table 1).

Table 1. Number of isolates (n) and contribution (\%) per center - MYSTIC Program Brazil 2002

\begin{tabular}{ccr}
\hline Center & $\mathbf{N}$ & \% \\
\hline 1 & 100 & 19.9 \\
2 & 70 & 13.9 \\
3 & 69 & 13.7 \\
4 & 76 & 15.1 \\
5 & 24 & 4.8 \\
6 & 52 & 10.3 \\
7 & 112 & 22.3 \\
Total & $\mathbf{5 0 3}$ & $\mathbf{1 0 0}$ \\
\hline
\end{tabular}

$\underline{\text { Isolates }}$

Five hundred and three Gram-negative bacilli responsible for the infections, based on the judgment of the investigators, were randomly selected at each center for this study. Multiple isolates of the same species from a single origin (same patient) were excluded. Non-sterile samples were submitted to semi-quantitative/quantitative cultures (catheter, bronchoalveolar lavage). Each participating laboratory identified the microorganisms. The central laboratory(Fleury-Medical Diagnostic Center) confirmed theidentification through conventional biochemical methodology or with the Vitek automated system.

\section{$\underline{\text { Susceptibility tests }}$}

The central laboratory determined the minimum inhibitory concentrations (MICs) of meropenem, imipenem, ciprofloxacin, ceftazidime, cefepime, cefotaxime, piperacillin/tazobactam, gentamicin, and tobramycin by E-test methodology (AB Biodisk, Solna, Sweden), and interpretations were made according to National Committee for Clinical Laboratory Standards [16]. Control strains, E. coli (ATCC 25922), E. coli (ATCC 35218), and Pseudomonas aeruginosa (ATCC 27853), were tested with each set of MIC determinations.

\section{$\underline{\text { Screening for extended spectrum } \beta \text {-lactamase (ESBL) }}$}

Escherichia coli and K. pneumoniae with MICs $\geq 2 \mu \mathrm{g} / \mathrm{mL}$ to any cephalosporins were submitted to the ESBL production test by double-disk synergy with amoxicillin/clavulanic acid and ceftazidime, ceftriaxone, cefotaxime, and aztreonam. Isolates with enhanced zones for any of the above-mentioned agents or for amoxicillin/clavulanic acid were considered ESBL producers for the purpose of this report; this test is not recommended by the NCCLS for confirmation of ESBL production. Control strains K. pneumoniae (ATCC 700603 - ESBL positive) and E. coli (ATCC 25922 - ESBL negative) were assayed with each test set.

\section{Results}

\section{$\underline{\text { Isolates }}$}

The prevalence of the isolated microorganisms is shown in Table 2. Pseudomonas aeruginosa (33\%) 
was the most frequent isolate, followed by $A$. baumannii (17.1\%), K. pneumoniae (12.1\%), E. coli (10.5\%), and E. cloacae (7.9\%).

Table 2. Prevalence of isolated microorganisms

\begin{tabular}{lcr}
\hline Microorganism & n & \% \\
\hline P. aeruginosa & 166 & 33.0 \\
A. baumannii & 86 & 17.1 \\
K. pneumoniae & 61 & 12.1 \\
E. coli & 53 & 10.5 \\
E. cloacae & 40 & 7.9 \\
S. marcescens & 28 & 5.6 \\
P. mirabilis & 16 & 3.2 \\
S. maltophilia & 11 & 2.2 \\
A. calcoaceticus & 7 & 1.4 \\
B. cepacia & 7 & 1.4 \\
Others & 27 & 5.6 \\
Total & $\mathbf{5 0 3}$ & $\mathbf{1 0 0}$ \\
\hline
\end{tabular}

$\underline{\text { Sample sources }}$

Table 3 shows the sample source distribution, with the most frequent samples being from blood/catheters (39.2\%), followed by respiratory $(25.7 \%)$ and urinary tracts $(16.7 \%)$. Table 4 shows the frequency of microorganisms per sample source.

Table 3. Sample source distribution (\%)

\begin{tabular}{lcr}
\hline Sample & N & \% \\
\hline Blood/catheter & 197 & 39.2 \\
Respiratory tract & 129 & 25.7 \\
Urinary tract & 84 & 16.7 \\
Skin/soft tissue & 33 & 6.5 \\
Others & 60 & 11.9 \\
Total & $\mathbf{5 0 3}$ & $\mathbf{1 0 0}$ \\
\hline
\end{tabular}

\section{$\underline{\text { Susceptibility patterns }}$}

Table 5 shows the overall results of susceptibility pattern of $P$. aeruginosa, A. baumannii, $K$. pneumoniae, and E. coli.

\section{Pseudomonas aeruginosa}

Pseudomonas aeruginosa $(\mathrm{n}=166)$ isolates presented susceptibility rates of $67.5 \%$ to piperacillin/tazobactam $\left(\mathrm{MIC}_{50} 24 \mu \mathrm{g} / \mathrm{mL}\right), 59.8 \%$ to meropenem $\left(\mathrm{MIC}_{50} 0.75\right.$ $\mu \mathrm{g} / \mathrm{mL}$ ), $57.3 \%$ to imipenem ( $\mathrm{MIC}_{50} 2 \mu \mathrm{g} / \mathrm{mL}$ ), $55.4 \%$ to cefepime $\left(\mathrm{MIC}_{50} 4 \mu \mathrm{g} / \mathrm{mL}\right)$, and $53.6 \%$ to ciprofloxacin $\left(\mathrm{MIC}_{50} 16 \mu \mathrm{g} / \mathrm{mL}\right)$. Ceftazidime, gentamicin and tobramycin presented susceptibility rates of $<50 \%$.

Susceptibility of $P$. aeruginosa by center is described in Table 6, with susceptibility rates shown for all six ICUs. Center 5 is not shown due to the small number of isolates of $P$. aeruginosa $(\mathrm{n}=5)$.

\section{A. baumannii}

A. baumannii $(\mathrm{n}=86)$ isolates presented susceptibility rates to meropenem of $89.5 \%$ ( $\left.\mathrm{MIC}_{50} 1 \mu \mathrm{g} / \mathrm{mL}\right), 88.4 \%$ to imipenem ( $\mathrm{MIC}_{50} 0.75 \mu \mathrm{g} / \mathrm{mL}$ ), and $74.4 \%$ to tobramycin $\left(\mathrm{MIC}_{50} 1.5 \mu \mathrm{g} / \mathrm{mL}\right)$. All remaining antimicrobials gave considerably lower susceptibility rates $(<55 \%)$.

\section{K. pneumoniae}

Among K. pneumoniae $(\mathrm{n}=61)$ isolates, all were susceptible to imipenem and meropenem $\left(\mathrm{MIC}_{90} 0.25\right.$ and $0.094 \mu \mathrm{g} / \mathrm{mL}$, respectively), with susceptibility rates of $86.9 \%$ to piperacillin/tazobactam and $72.1 \%$ to ciprofloxacin. Susceptibility to cephalosporins was $62.3 \%$, due to ESBL production in 23/61 (37.7\%).

E. coli

Among E. coli $(\mathrm{n}=53)$ isolates, all were susceptible to imipenem and meropenem $\left(\mathrm{MIC}_{90} 0.25\right.$ and 0.032 $\mu \mathrm{g} / \mathrm{mL}$, respectively), with susceptibility rates of $98.1 \%$ to piperacillin/tazobactam and $75.5 \%$ to ciprofloxacin. Susceptibility to cephalosporins was $86.8 \%$, due to ESBL production in 7/53 (13.2\%).

\section{Discussion}

The MYSTIC Program has generated a large data set for nosocomial isolates, with information on their 
Table 4. Frequency of microorganisms per sample source

\begin{tabular}{|c|c|c|c|c|}
\hline \multirow[b]{2}{*}{ Microorganism } & \multicolumn{4}{|c|}{$\mathbf{N}(\%)$} \\
\hline & Blood/Catheter & Respiratory tract & Urinary tract & Soft tissue \\
\hline P. aeruginosa & $44(22.3)$ & $52(40.3)$ & $31(36.9)$ & $19(57.6)$ \\
\hline A. baumannii & $41(20.8)$ & $22(17.1)$ & $16(19.0)$ & $3(9.1)$ \\
\hline K. pneumoniae & $27(13.7)$ & $12(9.3)$ & $10(11.9)$ & $3(9.1)$ \\
\hline E. coli & $19(9.6)$ & $5 \quad(3.9)$ & $14(16.7)$ & $3(9.1)$ \\
\hline E. cloacae & $22(11.2)$ & $10 \quad(7.8)$ & $2(2.4)$ & $3(9.1)$ \\
\hline S. marcescens & $14(7.1)$ & $9 \quad(7.0)$ & $1 \quad(1.2)$ & $1(3.0)$ \\
\hline Others & $30(15.2)$ & $19(14.6)$ & $10(11.9)$ & $1(3.0)$ \\
\hline Total n (\%) & $197(39.2)$ & $129(25.7)$ & 84 (16.7) & $33(6.5)$ \\
\hline
\end{tabular}

MICs. The data aggregated in our study was collected consecutively from patients hospitalized in ICUs in seven centers in Brazil during the 2002 edition of the MYSTIC Program. The program's main objective was toevaluate the susceptibility pattern of Gram-negative bacilli isolated from nosocomial infections. This is in accordance with the fundamentals of other microbiological surveillance studies, since these studies aim to identify regional patterns of resistance in specific settings. Surveillance programs also play a role as major contributors for guiding empirical antimicrobial therapy $[8,9]$. However, these programs are limited in their ability to answer all relevant clinical and microbiological outcome issues for all world regions, thus reinforcing the need for regional data.

Pseudomonas aeruginosa was identified in 33\% of the isolates, followed by A. baumannii (17.1\%), K. pneumoniae (12.1\%), E. coli $(10.5 \%)$ and $E$. cloacae $(7.9 \%)$. The frequencies of $P$. aeruginos $a$ and A. baumannii have risen significantly, when compared to the first MYSTIC edition in Brazil [19], but they have remained constant since the previous edition in 2001 [20]. This is probably due to the inclusion of new centers in the 2001 and 2002 editions and to the exclusive isolation of Gram-negative bacteria during both years. It should also be noted that, on the one hand, at least $55.9 \%$ of samples in the current edition were from clinically significant sources, either due to presumed sterility or to quantitative methods (blood, catheter and urinary tract). On the other hand, 25.7\% of the samples were from the respiratory tract, although always considered by investigators as causative agents of the infectious processes. Nevertheless, one cannot rule out completely the contribution of colonizers as part of the group of isolates. However, we believe that our study closely reflects the prevalence of Gramnegative bacteria causing nosocomial infections in the units that we evaluated. The high number of isolates from blood/catheters was expected, since we did not aim at establishing the prevalence of nosocomial infections. We concentrated on isolating clinically significant bacteria causing the infectious processes.

The susceptibility patterns detected by the MYSTIC Program in these seven Brazilian centers demonstrated resistance rates somewhat higher than the ones determined by other studies $[4,6,7,12,17,18]$. Previous editions of the MYSTIC Program in Brazil reported $P$. aeruginosa and $A$. baumannii sensitivities to carbapenems of $79 \%-82 \%$ and $85 \%-86 \%$, respectively $[19,20]$. Possible reasons for the higher resistance patterns observed in our study edition could be based on the program's selection of carbapenem user hospital units and of specialized centers, particularly intensive care units (ICUs). Other possible reasons could also be that all the centers but one were located in the south and southeast region of the country, which may reflect a specific influence of demographic characteristics of ICUs in the high resistance rates obtained. Furthermore, clonal spread among $P$. aeruginosa and $A$. baumannii within specific regions 
Table 5. Susceptibility patterns of E. coli, K. pneumoniae, A. baumannii, and P. aeruginosa - MYSTIC Program Brazil 2002

\begin{tabular}{|c|c|c|c|c|c|}
\hline \multirow[b]{2}{*}{ Species/antimicrobial } & \multicolumn{3}{|c|}{$\%$} & \multicolumn{2}{|c|}{$\mu g / m L$} \\
\hline & $\mathbf{S}$ & I & $\mathbf{R}$ & $\mathrm{MIC}_{50}$ & $\mathrm{MIC}_{90}$ \\
\hline \multicolumn{6}{|l|}{ P. aeruginosa $(\mathrm{n}=166)$} \\
\hline Cefepime & 55.4 & 20.5 & 24.1 & 4 & $>256$ \\
\hline Ceftazidime & 31.4 & 12.8 & 55.8 & $>192$ & $>256$ \\
\hline Imipenem & 57.3 & 6.6 & 36.1 & 2 & $>32$ \\
\hline Meropenem & 59.8 & 7.1 & 33.1 & 0.75 & $>32$ \\
\hline Piperacillin/tazobactam & 67.5 & 2.4 & 30.1 & 24 & $>256$ \\
\hline Ciprofloxacin & 53.6 & 10.2 & 36.2 & 16 & $>32$ \\
\hline Gentamicin & 47.6 & 0.0 & 52.4 & 16 & $>256$ \\
\hline Tobramycin & 44 & 0.6 & 55.4 & 12 & $>256$ \\
\hline \multicolumn{6}{|l|}{ A. baumannii $(\mathrm{n}=86)$} \\
\hline Cefepime & 31.4 & 37.2 & 31.4 & 64 & $>256$ \\
\hline Ceftazidime & 31.4 & 12.8 & 55.8 & $>128$ & $>256$ \\
\hline Imipenem & 88.4 & 1.2 & 10.4 & 0.75 & $>32$ \\
\hline Meropenem & 89.5 & 0.0 & 10.5 & 1 & $>32$ \\
\hline Piperacillin/tazobactam & 40.7 & 12.8 & 46.5 & 64 & $>256$ \\
\hline Ciprofloxacin & 31.4 & 12.8 & 55.8 & 32 & $>32$ \\
\hline Gentamicin & 53.5 & 10.5 & 36 & 4 & $>256$ \\
\hline Tobramycin & 74.4 & 7 & 18.6 & 1.5 & $>256$ \\
\hline \multicolumn{6}{|l|}{ K. pneumoniae $(\mathrm{n}=61)$} \\
\hline Cefepime & 62.3 & 0.0 & 37.7 & 0.094 & $>32$ \\
\hline Ceftazidime & 62.3 & 0.0 & 37.7 & 0.25 & $>32$ \\
\hline Cefotaxime & 62.3 & 0.0 & 37.7 & 0.125 & $>256$ \\
\hline Imipenem & 100 & 0.0 & 0.0 & 0.19 & 0.25 \\
\hline Meropenem & 100 & 0.0 & 0.0 & 0.032 & 0.094 \\
\hline Piperacillin/tazobactam & 86.9 & 4.9 & 8.2 & 2 & $>256$ \\
\hline Ciprofloxacin & 72.1 & 6.5 & 21.4 & 0.023 & $>32$ \\
\hline Gentamicin & 65.6 & 3.4 & 31 & 0.75 & $>256$ \\
\hline Tobramycin & 60.7 & - & 39.3 & 0.75 & $>256$ \\
\hline \multicolumn{6}{|l|}{ E. $\operatorname{coli}(n=53)$} \\
\hline Cefepime & 86.8 & 0.0 & 13.2 & 0.032 & $>32$ \\
\hline Ceftazidime & 86.8 & 0.0 & 13.2 & 0.125 & $>32$ \\
\hline Cefotaxime & 86.8 & 0.0 & 13.2 & 0.047 & $>256$ \\
\hline Imipenem & 100 & 0.0 & 0.0 & 0.19 & 0.25 \\
\hline Meropenem & 100 & 0.0 & 0.0 & 0.016 & 0.032 \\
\hline Piperacillin/tazobactam & 98.1 & 0.0 & 1.9 & 1 & 2 \\
\hline Ciprofloxacin & 75.5 & 0.0 & 24.5 & 0.008 & $>32$ \\
\hline Gentamicin & 84.9 & 0.0 & 15.1 & 0.5 & 48 \\
\hline Tobramycin & 86.7 & 0.0 & 13.2 & 0.5 & 32 \\
\hline
\end{tabular}


Table 6. Susceptibility of $P$. aeruginosa (\%S) per center - MYSTIC Program Brazil 2002

\begin{tabular}{lrrrrrrrr}
\hline & \multicolumn{7}{c}{ \% Susceptible } \\
\cline { 2 - 8 } & C & CFT & \multicolumn{1}{c}{ I } & \multicolumn{1}{c}{ M } & P/T & CPR & G & T \\
\hline Center 1 & 24.3 & 16.2 & 24.3 & 27 & 29.7 & 21.6 & 24.3 & 16.2 \\
Center 2 & 95.2 & 85.7 & 100 & 100 & 100 & 85.7 & 81 & 81 \\
Center 3 & 62.5 & 75 & 87.5 & 87.5 & 87.5 & 31.2 & 50 & 43.8 \\
Center 4 & 50 & 50 & 45 & 45 & 55 & 35 & 45 & 35 \\
Center 5* & -- & -- & -- & -- & -- & -- & -- & - \\
Center 6 & 40.9 & 40.9 & 36.4 & 36.4 & 59.1 & 31.8 & 27.3 & 27.3 \\
Center 7 & 75.6 & 75.6 & 75.6 & 82.2 & 88.9 & 62.2 & 66.7 & 66.7 \\
\hline Total & $\mathbf{5 5 . 4}$ & $\mathbf{3 1 . 4}$ & $\mathbf{5 7 . 3}$ & $\mathbf{5 9 . 8}$ & $\mathbf{6 7 . 5}$ & $\mathbf{5 3 . 6}$ & $\mathbf{4 7 . 6}$ & $\mathbf{4 4}$ \\
\hline C =c.5
\end{tabular}

$\mathrm{C}=$ cefepime;CFT =ceftazidime; I=imipenem; $\mathrm{M}=$ meropenem; $\mathrm{P} / \mathrm{T}=$ piperacillin/tazobactam; $\mathrm{CPR}=$ ciprofloxacin; $\mathrm{G}=$ gentamicin; $\mathrm{T}=$ tobramycin. * Center 5 - not listed, only 5 isolates.

was detected and is currently under more extensive evaluation by investigators (CK, CM).

Pseudomonas aeruginosa $(\mathrm{n}=166)$ isolates had quite high resistance rates against all antimicrobials, with descending order of susceptibility rates of $67.5 \%$ to piperacillin/tazobactam $\left(\mathrm{MIC}_{50} 24 \mu \mathrm{g} / \mathrm{mL}\right), 59.8 \%$ to meropenem $\left(\mathrm{MIC}_{50} 0.75 \mu \mathrm{g} / \mathrm{mL}\right.$ ), and $57.3 \%$ to imipenem $\left(\mathrm{MIC}_{50} 2 \mu \mathrm{g} / \mathrm{mL}\right)$. Susceptibility of $P$. aeruginosa varied greatly among the centers, with rates ranging from $100 \%$ susceptible for carbapenems and piperacillin/tazobactam in center 2 to $<30 \%$ susceptible for all antimicrobials in center 1 . As previously mentioned, clonal spread may have contributed to the susceptibility observed in specific centers. On the other hand, A. baumannii $(\mathrm{n}=86)$ isolates presented susceptibility rates to meropenem of $89.5 \%\left(\mathrm{MIC}_{50}\right.$ $1 \mu \mathrm{g} / \mathrm{mL}$ ), $88.4 \%$ to imipenem ( $\mathrm{MIC}_{50} 0.75 \mu \mathrm{g} / \mathrm{mL}$ ), and $74.4 \%$ to tobramycin $\left(\mathrm{MIC}_{50} 1.5 \mu \mathrm{g} / \mathrm{mL}\right)$, which is in accordance with previous MYSTIC editions in our country [19,20]. Escherichia coli ESBLproducing isolates $(13.2 \%)$ presented prevalences similar to those of previous editions of this study [19,20]. However, K. pneumoniae ESBL-producing isolates presented lower prevalence rates $(37.7 \%)$, when compared to the previous edition $(63.5 \%$ in the 2001 edition) [20]. Tendency analysis will be performed with future editions of the program, in order to check this finding.
Additionally, results from the 2002 edition of the MYSTIC Program in Brazil, Colombia, Peru and Venezuela were also used in the international program for pharmacodynamic comparisons, based on Monte Carlo simulation methods [21]. This program, named OPTAMA, identified differences in pharmacodynamic target attainment for commonly-used antimicrobials in South America. It also showed some discordance with percent susceptibility for certain agents, meaning that the use of pharmacodynamic target attainment may be a more accurate predictor of microbiological success. Thus, we concluded that: a) any of the carbapenems or cephalosporins would be appropriate empirical therapy when $E$. coli is suspected; b) the carbapenems should be the agents of choice for suspected $K$. pneumoniae infections; c) since no single regimen had high target attainment against $A$. baumannii and $P$. aeruginosa, the use of combination therapy to treat these pathogens in South America may be justified.

In conclusion, resistance development to antimicrobials is currently a major concern for the medical community worldwide, since infections caused by resistant bacteria seems to be associated with worsened morbidity factors (hospitalization, death and illness rates) [22]. The implementation of monitoring programs is an important part of the preventative strategy against progression of resistance. Surveillance in ICUs apparently offers a unique opportunity to detect 
the emergence of resistance in bacteria used as sentinel agents, especially in units with high antibiotic usage densities $[1,23]$. Our study confirms previous findings that carbapenem resistance among Enterobacteriacea is still rare in this region $[4,6,7,12,17-20]$. On the other hand, A. baumannii and $P$. aeruginosa have become particularly problematic organisms in Brazil, because of their prevalence and resistance patterns. Since $A$. baumannii and $P$. aeruginosa play an important role in nosocomial infections in this environment, added to the fact that they were not highly susceptible to any of the drugs, and because no single regimen had high target attainment in data generated with a Monte Carlo simulation program using the same data [21], the use of empirical combination therapy to treat these pathogens may be justified.

\section{MYSTIC Study Group Brazil}

Jorge Sampaio, André Hsiung, Marcelo Marotti, Jorge B. Amarante, Hélio Sader, Suzane Silbert, Cássia M. Zocolli, Jorge D. de Mattos, Beatriz M. Moreira, Marinês D. Martino, Luis F. Camargo, Adília Segura, Julival Ribeiro, Lycia Mimica, Sueli Ykko.

\section{References}

1. Mendes C., Turner P.J. Unit differences in pathogen occurrence among European MYSTIC Program (19972000). Diagnostic Microbiology and Infectious Diseases 2001;41:191-6.

2. Archibald L., Phillips D., Monnet D., et al. Antimicrobial resistance in isolates from inpatients and outpatients in the United States: increasing importance of the intensive care unit. Clin Infect Dis 1997;24:211-5.

3. Jones R.N. The emergent needs for basic research, education, and surveillance of antimicrobial resistance. Problems facing the report from the American Society for Microbiology Task Force on Antibiotic Resistance. Diagnostic Microbiology and Infectious Diseases 1996; $25: 153-61$.

4. Jones R.N., Pfaller M.A. Bacterial resistance: a worldwide problem. Diagnostic Microbiology and Infectious Diseases 1998;31:379-88.
5. Pfaller M.A., Jones R.N., Biedenbach D.J. MYSTIC Program Study Group (USA). Antimicrobial resistance trends in carbapenem prescribing medical units: report of the 1999 and 2000 results from MYSTIC Program (USA). Diagnostic Microbiology and Infectious Diseases 2001;41(4):177-82.

6. Diekema D.J., Pfaller M.A., Jones R.N., et al. Survey of bloodstream infection due to Gram-negative bacilli: frequency of occurrence and antimicrobial susceptibility of isolates collected in the United States, Canada and Latin America for the SENTRY Antimicrobial Surveillance Program. 1997. Clin Infect Dis 1997;29:595-607.

7. Diekema D.J., Pfaller M.A., Jones R.N., et al. Trends in antimicrobial susceptibility of bacterial pathogens isolated from patients with bloodstream infection in the USA, Canada and Latin America. SENTRY Participants Group. Int J Antimicrob Agents 2000;13:257-71.

8. Jones R.N. Detection of emerging resistance patterns within longitudinal surveillance systems: data sensitivity and microbial susceptibility. J Antimicrob Chemother 2000;46(topic T2):1-8.

9. Masterton R.G. Surveillance studies: how can they help the management of infection? J Antimicrob Chemother 2000;46(topic T2):53-8.

10. Jones R.N. Contemporary antimicrobial susceptibility patterns of bacterial pathogens commonly associated with febrile patients with neutropenia. Clin Infect Dis 1999;29:495-502.

11. Pfaller M.A., Jones R.N., Doern G.V., Kugler K.C. Bacterial pathogens isolated from patients with bloodstream infection: frequencies of occurrence and antimicrobial susceptibility patterns from the SENTRY Antimicrobial Resistance Surveillance Program (United States and Canada). Antimicrob Agents Chemother 1998;42:1762-70.

12. Sader H.S., Jones R.N., Winokur P.L. Antimicrobial susceptibility of bacteria causing urinary tract infections in Latin American hospitals: results from the SENTRY Antimicrobial Surveillance Program (1997). Clin Microbiol Infect 1999;5:478-87.

13. Jones R.N., Masterton R. Establishing the value of antimicrobial surveillance programs. Diagnostic Microbiology and Infectious Diseases 2001;41:171-5.

14. Turner P.J. MYSTIC (Meropenem Yearly Susceptibility est Information Collection): a global overview. J Antimicrob Chemother 2000;46(Topic T2):9-23.

15. Rhomberg P.R., Jones, R.N., The MYSTIC Program (USA) Study Group. (2003). Antimicrobial spectrum of activity for meropenem and nine broad spectrum antimicrobials: report from the MYSTIC Program (2002) in North America. Diagn Microbiol Infect Dis 2002;47:365-72.

16. NCCLS. Performance Standards for Antimicrobial Susceptibility Testing: eleventh informational supplement M100-S13. NCCLS, Wayne, Pa, 2003. 
17. Jones R.N. Resistance Patterns Among Nosocomial Pathogens: Trends Over the Past Few Years. Chest 2001;119(2 Suppl):397S-404S.

18. Sader H.S., Jones R.N., Gales A.C., et al. Antimicrobial susceptibility patterns for pathogens isolated from patients in Latin American medical centers with a diagnosis of pneumonia: analysis of results from the SENTRY Antimicrobial Surveillance Program (1997): SENTRY Latin America Study Group. Diagn Microbiol Infect Dis 1998;32:289-301.

19. Mendes C., Hsiung A., Kiffer C., et al. Evaluation of in vitro Activity of 9 Antimicrobials Against Bacterial Strains Isolated From Patients in Intensive Care Units in Brazil: MYSTIC Antimicrobial Surveillance Program. Braz J Inf Dis 2000;4(5):236-44.

20. Mendes C., Oplustil C., Turner P., et al. Antimicrobial susceptibility in intensive care units: MYSTIC Program Brazil 2001. XI Congreso Panamericano de Infectología, 11-14 May, Córdoba, Argentina, 2003.

21. Kiffer C.R.V., Mendes C., Kuti J.L., Nicolau D.P. The OPTAMA Program, South America 2002: Pharmacodynamic Comparisons of Antimicrobials Against Nosocomial Isolates of E. coli, K. pneumoniae, A. baumannii and P. aeruginosa. XXII Congresso Brasileiro de Microbiologia, Florianópolis, Brazil, 17-20 November, 2003 (IH-101).

22. Scheld W.M. Maintaining fluoroquinolone class efficacy: review of influencing factors. Emerg Inf Dis 2003;9(1):1-9.

23. Pfaller M.A., Jones R.N., Biedenbach D.J. MYSTIC Program Study Group (USA). Antimicrobial resistance trends in carbapenem prescribing medical units: report of the 1999 and 2000 results from MYSTIC Program (USA). Diagnostic Microbiology and Infectious Diseases 2001;41(4):177-82. 